\title{
Imaging Man's Weaknesses with Simple Harmonic Generation
}

\author{
Simon C. Watkins
}

Center for Biologic Imaging, Department of Cell Biology and Physiology, University of Pittsburgh School of Medicine, 3500 Terrace Street, Pittsburgh, PA

Vascular bioengineering encompasses multi-scale studies of the structural and mechanical properties of native and engineered soft tissues. Whereas traditional histological methods have been the mainstay of the research scientist dissecting the process of vascular failure in man, these methods rely on fixation, embedment and sectioning. The bioengineer must be able to generate quantitative data correlating mechanical characteristics with real time measures of structural change. These approaches must be performed on living in vivo or ex vivo specimens mounted in specialized chambers specifically designed to test vessel mechanics. Recently, a pressing need has arisen to combine these studies with optical correlates of damage or recovery. To this end, we use multiphoton imaging of endogenous molecular signals to provide temporal information regarding changes in structural features demarking vascular failure in abdominal and cerebral aneurysms. Elastin degradation occurs during the early stages of aneurysm development and characterization of this process can be used to monitor the initiation, growth, and rupture of the vascular wall. The nearinfrared excitation wavelengths used in multiphoton microscopy (MPE) permits the axial depth of penetration required for imaging optically thick segments of the vascular tissue. We combine MPE imaging of the intrinsic autofluorescence of elastin with second harmonic generation (SHG) imaging of collagen to provide fundamental information about vascular failure and/or regenerative potential (Figure 1 shows an example of a late stage aneurysm, this is a projection of a 200 micron image stack, elastin autofluorescence is shown in green, the second harmonic generation signal in red, the clear disruption of the elastin is evident in this image as is the lack of order of the collagen). Unlike fluorescence, SHG is a nonlinear optical process involving no excitation. Collagen is intrinsically noncentrosymmetric and emits SHG. Elastin fluorescence and SHG are monitored simultaneously (without the need for staining) and, following quantitative methods and posthoc image segmentation and 3D reconstruction, show how structural components become corrupt in disease. These studies demonstrate the ability to use the autofluorescence and SHG imaging capabilities of multiphoton microscopy to provide high-contrast images of elastin and collagen, respectively and thereby reveal critical information regarding disease development and progression at a microstructural level.

This work was supported by 1 U54 RR022241 (SCW). 
Figure 1: An example of a late stage aneurysm, this is a projection of a 200 micron image stack, elastin autofluorescence is shown in green, the second harmonic generation signal in red, the clear disruption of the elastin is evident in this image as is the lack of order of the collagen.

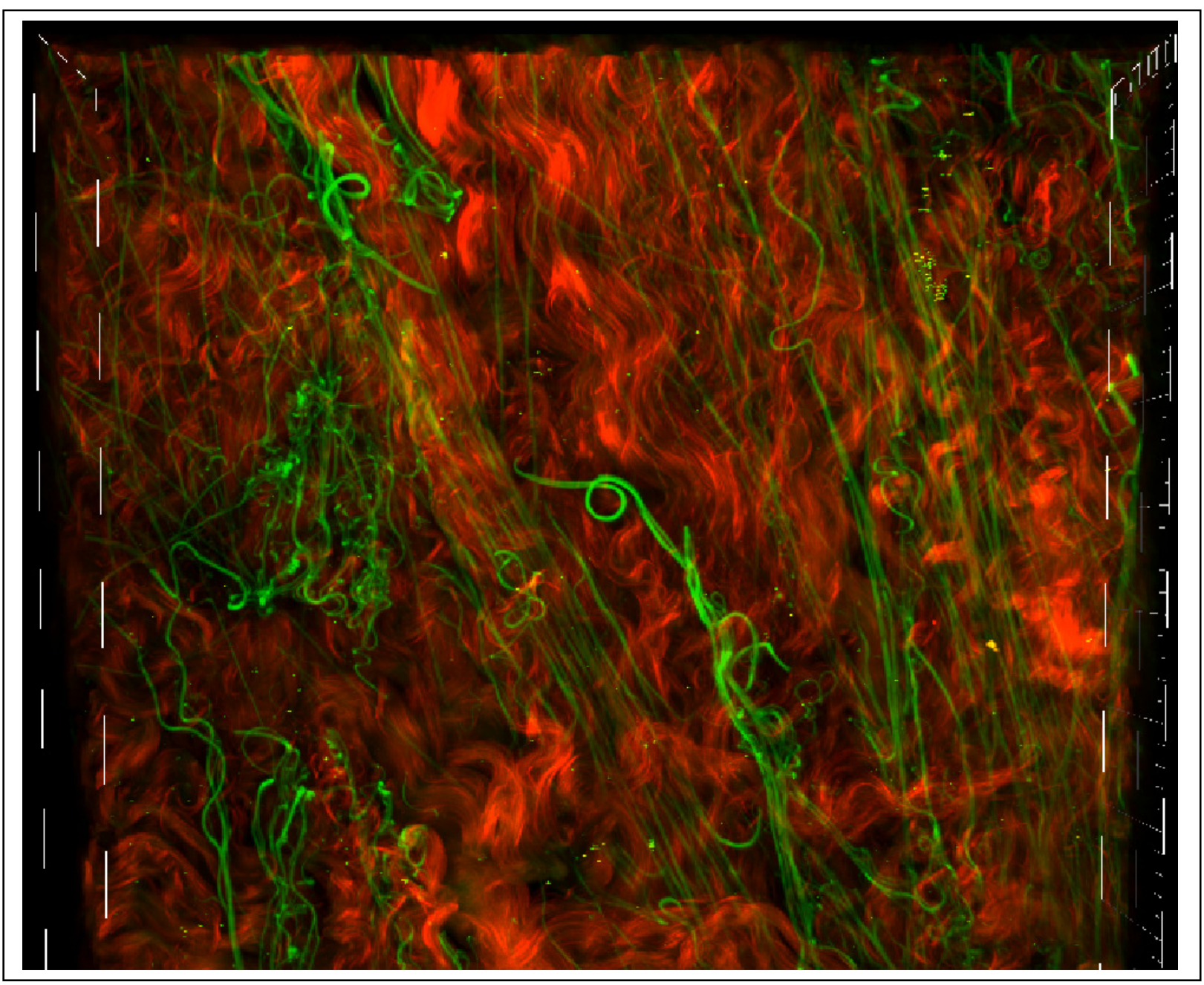

